\title{
Measurements of methane emissions from natural gas gathering facilities and processing plants: measurement methods
}

J. R. Roscioli et al.

Correspondence to: S. C. Herndon (herndon@aerodyne.com) 
Supplemental Information

\section{A. Using dual tracer ratio for a selection criterion}

Depending upon the outcome of the above $E / M$ and $R^{2}$ criteria, the plume is either rejected, analyzed using single-correlation, or is a candidate for dual area or dual correlation analysis. One of the advantages of using two tracers is that it ensures that a measured plume reflects the true facility level emission rate. Comparing the downwind ratio of the tracer concentrations:

$$
R_{\text {plume }}=\frac{[\operatorname{tracer} A]}{[\operatorname{tracer} B]}
$$

(determined using either correlation or area ratio analysis) to the known tracer flow rates:

$$
R_{\text {flow }}=\frac{f \operatorname{low}(A)}{f \operatorname{low}(B)}
$$

provides an immediate assessment of the legitimacy of the plume in assessing the FLER. That is, in cases where the plume tracer ratio significantly deviates from the known tracer flow ratio, the plume must be rejected. In this study, the plume tracer ratio must be within a factor of 1.5 of the flow rate (or a factor of 2 in the case of dual area analysis).

It should be noted that the relevant criterion is $0.67<R_{\text {plume }} / R_{\text {flow }}<1.5$ (i.e. $R_{\text {plume }}$ must be within a factor of 1.5 of $R_{\text {flow }}$ ), which is fundamentally different than a criterion of $+/-50 \%$. When using a factor (1.5), the order of the tracers in $R_{\text {plume }}$ or $R_{\text {flow }}$ is irrelevant to whether the plume is accepted. That is, whether the $R_{\text {flow }}$ and $R_{\text {plume }}$ are defined as $R_{i}=C_{2} H_{2} / N_{2} O$ or $\mathrm{N}_{2} \mathrm{O} / \mathrm{C}_{2} \mathrm{H}_{2}$ has no bearing on its final fate as an accepted or rejected plume. In a case where the 
relevant acceptance window is $+/-50 \%$, a plume may be accepted for $R_{i}=C_{2} H_{2} / N_{2} O$ but rejected if $\mathrm{R}_{\mathrm{i}}=\mathrm{N}_{2} \mathrm{O} / \mathrm{C}_{2} \mathrm{H}_{2}$. A histogram of $\mathrm{R}_{\text {plume }} / \mathrm{R}_{\text {flow }}$ for all dual tracer plumes (both accepted and rejected) in this campaign is shown in Figure 1 (using area analysis for $R_{\text {plume }}$ ). It is well described by a lognormal distribution:

$$
P(x)=\frac{1}{x \sigma \sqrt{2 \pi}} e^{-\frac{(\ln x-\mu)^{2}}{2 \sigma^{2}}}
$$

Such a distribution is expected when considering mathematical ratios, where the relevant value is the fractional deviation from unity. The resulting variance in this distribution reflects the inherent uncertainty in tracer release methods in the absence of any numerical or postacquisition selection criteria.

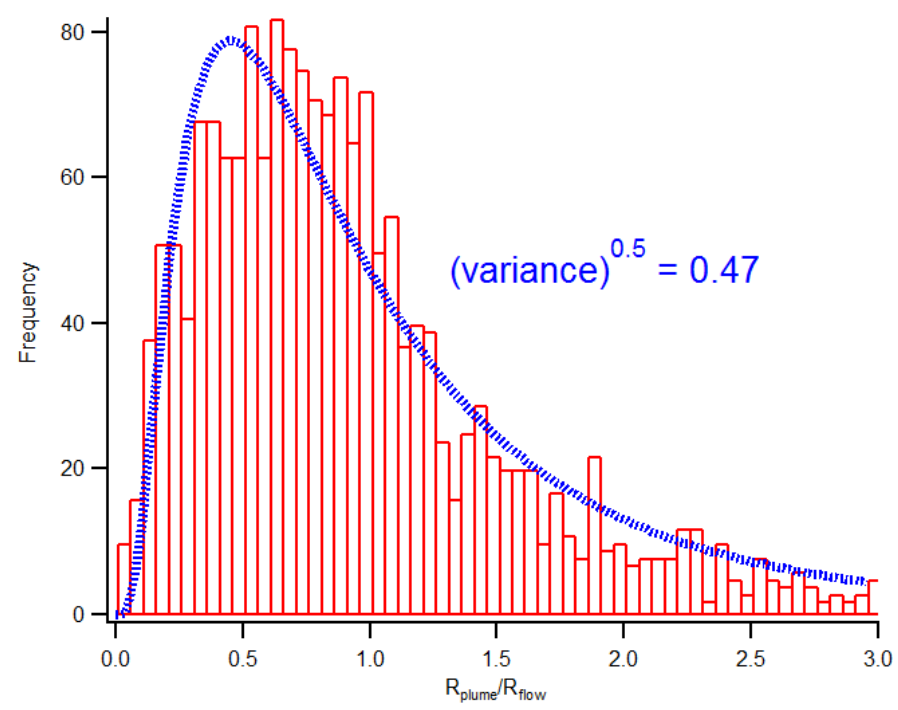

Figure 1. Histogram of $R_{\text {plume }} / R_{\text {flow }}$ for all dual tracer plumes, with fit to lognormal distribution (blue dashed line).

Uncertainty and weighting of individual types of plumes

The large number of plumes associated with this dataset (1833 accepted plumes), lends itself to a statistical analysis of each plume type (dual correlation, dual area, single correlation). 
This measured uncertainty can then provide a weighting to each plume type, when multiple types are obtained at a given facility. The resulting histograms are presented in Figure 2 for dual correlation (2(a)), dual area (2(b)), and two variants of single correlation: only single correlation plumes where the tracer ratio is within a factor of 2 of the known tracer flow rates (i.e. $\left.0.5<R_{\text {plume }} / R_{\text {flow }}<2\right)(2(c))$, and all single correlation plumes (2(d)). As is discussed in the Measurements paper,(Mitchell et al., 2014) the variances in these distributions are used to weight the FLER estimates obtained from each individual plume into a weighted average FLER, or WAFLER.
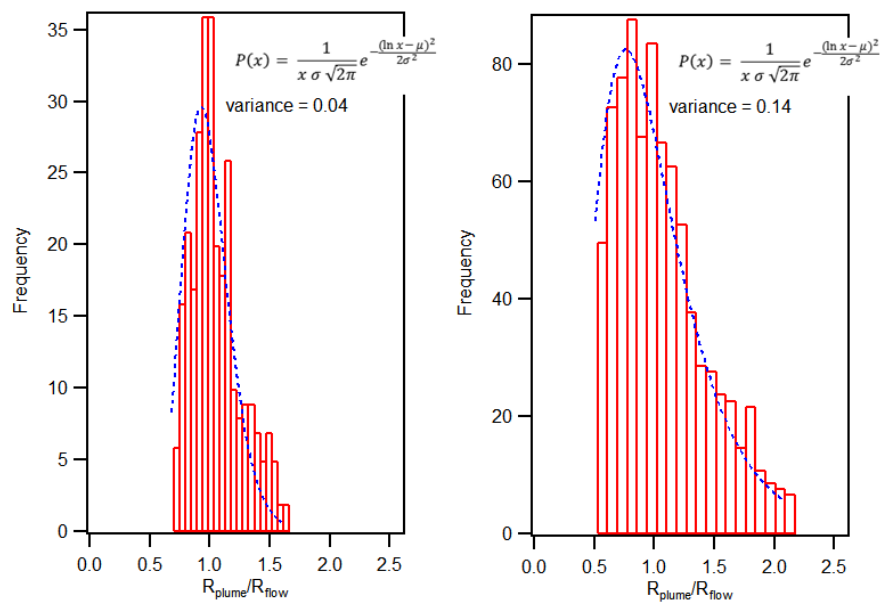

Figure 2. Histograms of errors associated with: (a) dual correlation plumes, (b) dual area plumes, (c) single correlation plumes in which the tracer ratio (by area) was within a factor of 1.5, and (d) all single correlation plumes (error was determined by correlation analysis of tracers). Blue dashed traces are lognormal fits to the distribution. Variance values are determined from the empirical distribution (not
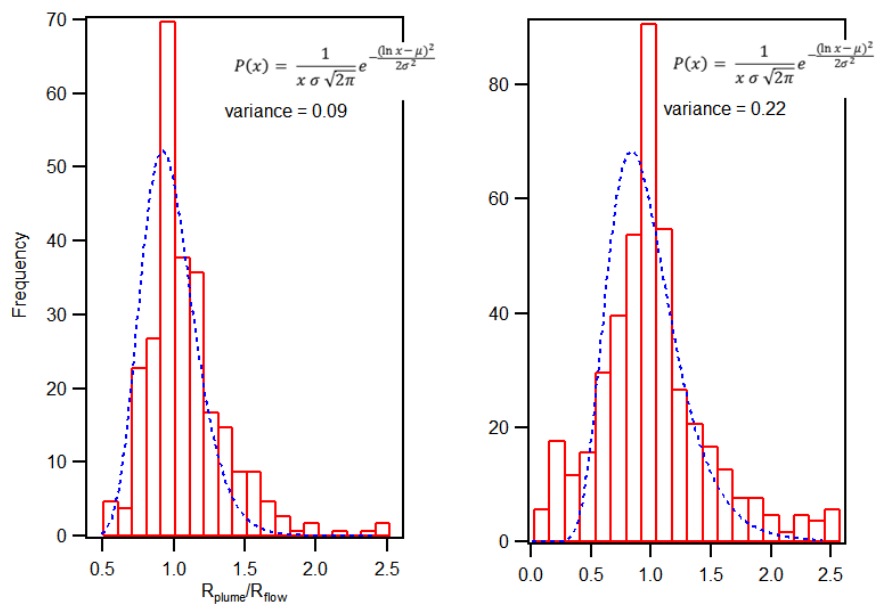
lognormal fit). 


\section{B. Decision Process for Plume Acceptance}

Figure 3 depicts the plume decision process used both accept and classify plume types during

the G\&P field campaign. Examples of dual correlation, dual area, and single correlation plumes are illustrated in Figures 2-5 in the main text of the report.

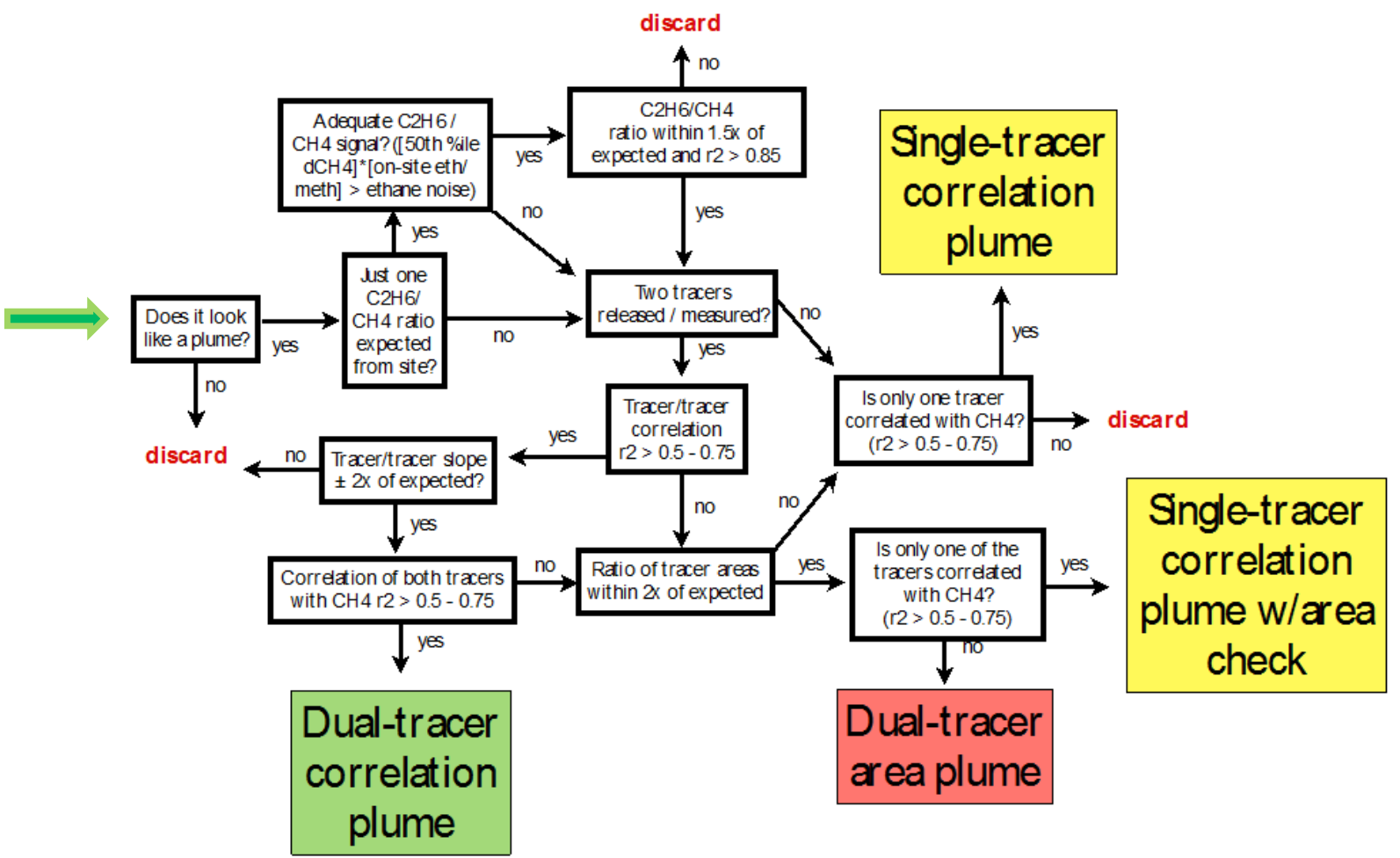

Figure 3. The systematic process by which all plume data were evaluated, showing that the analysis method assigned for each plume was based on established acceptance criteria. Plumes were discarded if the minimum acceptance criteria were not met. The analysis method of linear combination is not included as it was only employed to analyze one facility (facility \#118), for which the other three methods could not be used. In some cases, only one of the tracers is correlated with methane (as in a "single-tracer correlation" plume) but the ratio of integrated tracer areas is within $1.5 x$ of the expected tracer ratio. In such cases, facility-level methane emissions are calculated just as they are for "single tracer correlation" plumes, but the estimate is assigned a lower uncertainty, since the tracer area ratio provides an additional check on co-dispersion. 


\section{Lofted and Buoyant Plume Effects in Tracer release measurements}

During the G\&P field campaign, tracers were typically released at ground level ( $2 \mathrm{~m})$. While most $\mathrm{CH}_{4}$ emission sources are at ground level, some $\mathrm{CH}_{4}$ may be released in flares and exhaust stacks, where the elevated release position and buoyancy of the plume can result in the plume not being fully recovered at some downwind distances.

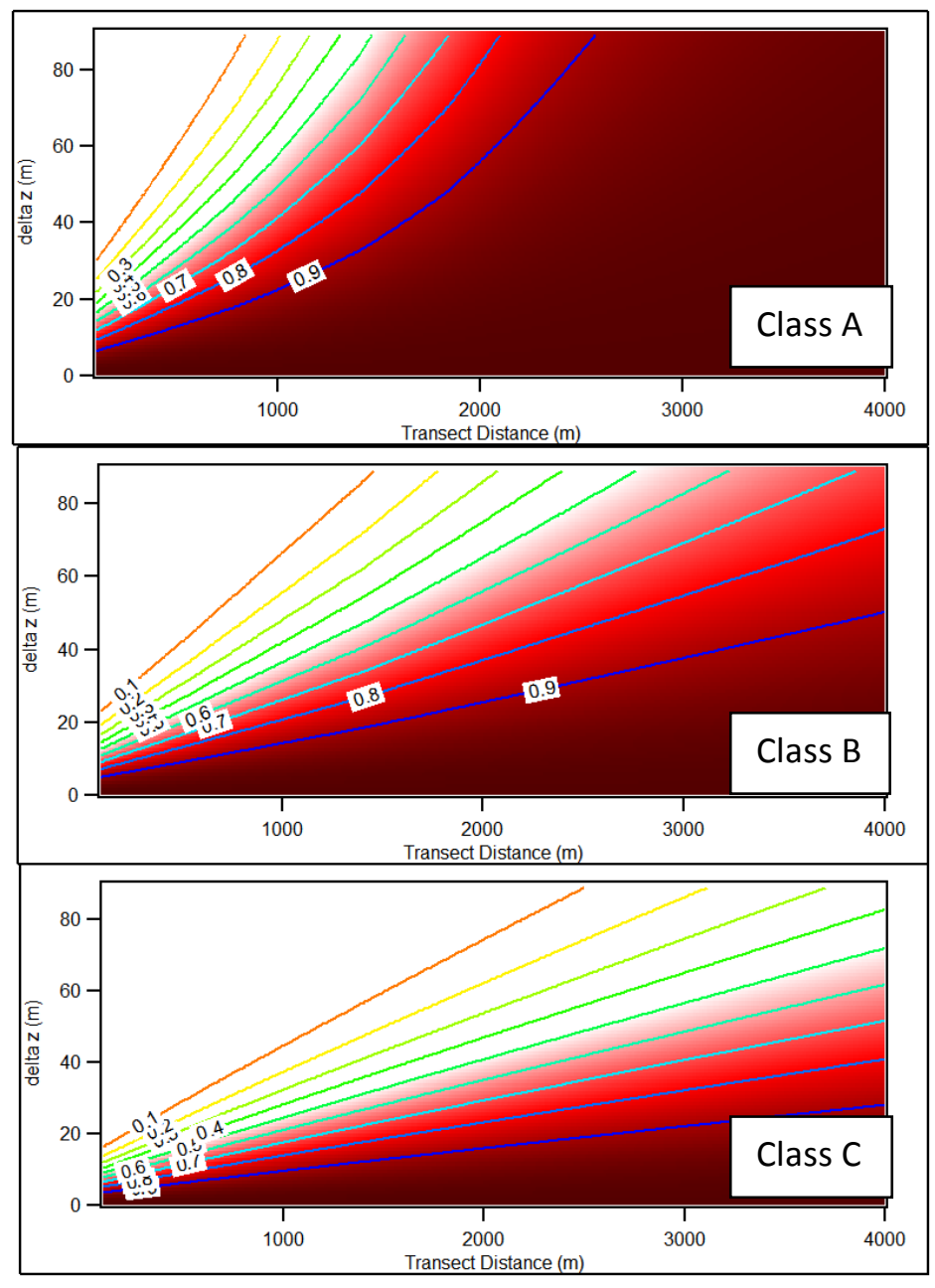

Figure 4. Fraction of $\mathrm{CH}_{4}$ emission recovered at ground level as a function of plume height (left axis) and downwind distance. Top, middle and bottom panels correspond to atmospheric stability classes $A, B$, and $C$, respectively.

\section{Plume Buoyancy and Vertical Rise}

The effect of large initial vertical velocities of $\mathrm{CH}_{4}$ from exhaust stacks can be modeled using the Brigg's Equations. A survey of gathering facility exhaust stack heights indicates that $\sim 7.5 \mathrm{~m}$ is typical for an exhaust stack height. Exhaust flow (mostly $\left.\mathrm{CO}_{2}\right)$ rates are highly variable, but 7500 CFM is $75^{\text {th }}$ percentile among a survey of several hundred compressor stations. Assuming an $800 \mathrm{~K}$ exhaust temperature, the maximum exhaust plume height can be anywhere from 25-50 m, 
depending upon the local wind conditions $(2-5 \mathrm{~m} / \mathrm{s})$.

\section{Gaussian plume modeling}

In the absence of plume buoyancy, the effect of an elevated plume and ground level tracer upon the FLER measurement will results in a low-biased measurement, according to Gaussian dispersion modeling.

The degree of this bias will depend upon the atmospheric stability class (A, B, or C in this case) and the height of the emission. Shown in Figure 4 are $32 \mathrm{D}$ plots of the bias as a

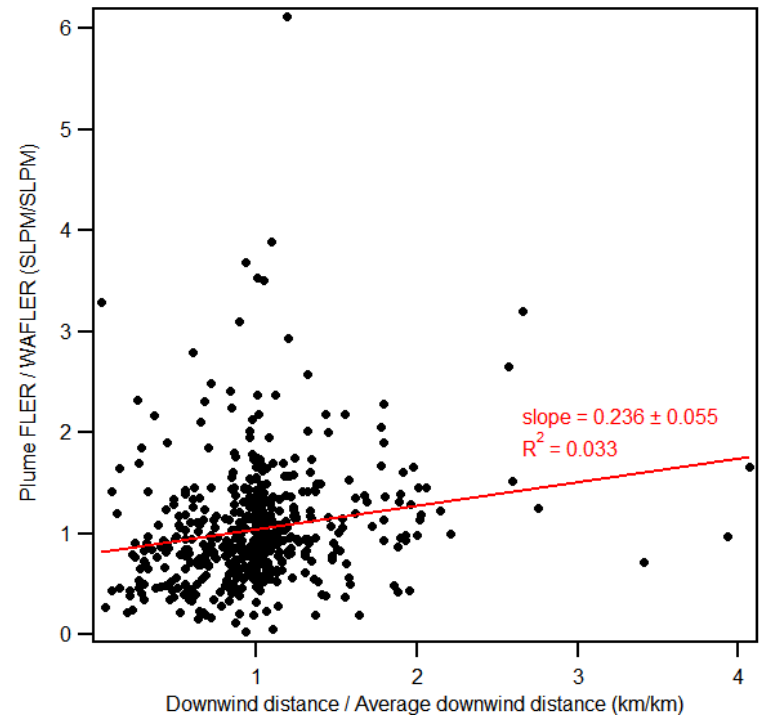

Figure 5. FLER values derived for individual plumes, normalized to their respective facility WAFLER, as a function of downwind distance, normalized to the average downwind distance at each facility.

function of downwind distance and vertical

distance between tracer and emissions source. Contour lines indicate the fractional bias of the tracer measurement for each stability class (i.e. $0.9=10 \%$ bias, $0.8=20 \%$ bias, etc.). When including the buoyancy and momentum effects described above, the overall $\Delta z$ can be $30-60 \mathrm{~m}$. This implies that significant fractions ( $\sim 50 \%$ ) of the $\mathrm{CH}_{4}$ emanating from the stack may not be recovered when downwind distances are $<1000 \mathrm{~m}$. The degree to which this affects the overall FLER estimate, considering the larger common $\mathrm{CH}_{4}$ emission sources on the ground, is discussed in the second part of this series.

In cases where elevated $\mathrm{CH}_{4}$ plumes are present, the elevated methane plume will extend to ground level as it travels downwind. If this vector is a significant contributor to the 
overall facility emission rate, the measured FLER will increase with downwind transect distance. Figure 5 displays the FLER derived from individual plumes obtained during the campaign, normalized by each site's measured WAFLER, as a function of downwind distance, normalized by average downwind distance at each site. If a systematic bias is present in the data due to contributions from elevated plumes, it will manifest as a positive correlation in the graph in Figure 5. As shown by the blue fit line, there is a slight positive slope to the fit, but the $R^{2}$ value of the fit is near zero (indicating nearly no correlation). While this is not necessarily a quantitative assessment of the effect of lofted plumes, it does suggest that it is not a major source of bias. The associated measurements paper addresses the role of these lofted emissions to the dataset from the perspective of modeled combustion emissions. 